\title{
Malignant ossifying fibromyxoid tumor of the calvaria: illustrative case
}

\author{
Jan T. Hachmann, MD, and R. Scott Graham, MD \\ Department of Neurologic Surgery, Virginia Commonwealth University Health System, Richmond, Virginia
}

BACKGROUND Ossifying fibromyxoid tumor (OFMT) is a rare entity of soft tissue tumor that most commonly occurs in the subcutaneous tissues of trunk or extremities with occasional cases involving the head and neck; however, primary involvement of the skull has not been reported. While historically considered slow-growing benign to intermediate malignant, few cases of atypical or malignant features have been described.

OBSERVATIONS Herein, the authors present a case of malignant OFMT with primary skull and transcranial extension. The tumor caused lytic calvarial destruction with intra- and extracranial soft tissue components. Gross total resection was performed, and histopathology revealed malignant OFMT with 40 mitoses per 50 high-power fields and moderate nuclear atypia.

LESSONS OFMT can rarely occur in the head and neck and, as reported herein, may involve the skull with intracranial extension. While no uniformly recognized histological criteria for malignancy exist, a three-tiered classification has been proposed: typical, atypical, and malignant, based on features such as hypercellularity, mitotic activity, infiltrative growth, and/or nuclear atypia. Malignant variants should be considered along the high-grade sarcoma spectrum with elevated risk for recurrence or metastatic spread. Routine adjuvant radiotherapy is not typically recommended; however, surveillance imaging is advised.

https://thejns.org/doi/abs/10.3171/CASE21346

KEYWORDS malignant ossifying fibromyxoid tumor; sarcoma; calvaria; skull; oncology; ossifying fibromyxoid tumor

Ossifying fibromyxoid tumor (OFMT) is a rare and distinct mesenchymal soft tissue tumor entity of unknown histogenesis. It was first described in 1989 by Enzinger et al. as "ossifying fibromyxoid tumor of soft parts." 1 While historically considered benign or indeterminate/ borderline malignant potential, frank malignant variants have been recognized and are considered part of the high-grade sarcoma spectrum. $^{2-14}$ While the exact differentiation cell line remains unknown, there is some evidence of putative Schwannian/neuroectodermal etiopathogenesis or myoepithelial/cartilaginous differentiation, $1,4,5,13,15,16$ with a "scrambled phenotype"10 being a leading hypothesis.

Most frequent affected regions by this tumor subtype include the subcutaneous soft tissues of the trunk and extremities, with proximal more common than distal locations. Occasional cases involving the head and neck region have been described, affecting mainly the anterior and posterior neck soft tissues, ${ }^{1-3,5,6,15,17-20}$ face, and oral cavity (e.g., cheek, lip, chin, soft palate, nose, zygoma/parotid, submental and submandibular region, and tongue). ${ }^{2,4,12,14,15,18-31}$ Rarely, involvement of the scalp has been described..$^{3,7,19,32}$ However, to our knowledge, this is the first reported case with primary calvarial bone involvement and transcranial extension.

\section{Illustrative Case}

The patient is a 25-year-old female with a medical history of polycystic ovarian syndrome and hypothyroidism who presented with gradually enlarging left scalp swelling over a period of 12 months. On exam, there was scalp expansion over the left temporoparietal region; the overlying skin was unremarkable without any overt changes. Prior to neurosurgical referral, the following workup had been performed: An ultrasound examination showed a cystic scalp mass with low-level internal echoes, foci of hyperechogenicity, internal vascularity, and thin hypervascular rim. A punch biopsy to the depth of the superficial subcutaneous fat showed only sparse perivascular lymphocytic inflammation and slight sclerosis of dermal collagen but was otherwise unremarkable. A plain skull radiograph was obtained that showed a $3-\mathrm{cm}$ radiolucent lesion at the left parietal calvaria. Magnetic resonance imaging (MRI) of the brain

ABBREVIATIONS CT = computed tomography; HPF = high-power field; MRI = magnetic resonance imaging; OFMT = ossifying fibromyxoid tumor. INCLUDE WHEN CITING Published August 23, 2021; DOI: 10.3171/CASE21346.

SUBMITTED June 13, 2021. ACCEPTED June 28, 2021.

(c) 2021 The authors, CC BY-NC-ND 4.0 (http://creativecommons.org/licenses/by-nc-nd/4.0/). 
without and with contrast was obtained, and the patient was referred for neurosurgical evaluation. Neurological exam was unremarkable. The MRI revealed a lytic transcranial left parietal mass lesion with intra- and extracranial soft tissue extension and lytic destruction of the calvarial skull in the area. Dimensions of the mass were $5 \mathrm{~cm}$ anteroposterior by $3.8 \mathrm{~cm}$ transverse by $5.6 \mathrm{~cm}$ craniocaudal. The intracranial component appeared limited by the dura. The cortical surface was depressed by approximately $1 \mathrm{~cm}$ without evidence of brain invasion or adjacent perifocal edema. The mass showed homogeneous intermediate signal intensity on T1and T2-weighted imaging, no diffusion restriction, and uniform avid contrast enhancement (Fig. 1).

The patient underwent an elective left parietal craniectomy and methylmethacrylate cranioplasty (Fig. 2). A linear incision was planned to span the lesion extending from normal inferior to normal superior bony margins; the incision was opened with close attention not to violate the capsule of the mass. The periosteum was carefully freed up and the temporalis muscle was partially reflected. Two burr holes were made and a craniectomy was performed circumferential to the lesion with a clear margin. The tumor was noted to be adherent to the underlying dura. A plane was carefully developed using blunt and sharp dissection. Gross total resection was performed en bloc, and the mass was sent for frozen and permanent pathology. After the frozen section revealed a potentially sarcomatous lesion, the underlying dura in contact with the lesion was excised with a margin and sent for permanent pathology. A dural allograft patch was then secured in a standard watertight fashion. A methylmethacrylate cranioplasty implant was shaped to size and form and was secured in place using standard cranial plating technique. Incision was closed in the standard fashion.

The outer surface of the skull was smooth with an attached irregularly shaped soft tissue mass measuring $6.8 \times 6.5 \times 2.7 \mathrm{~cm}$. The mass showed dense fibroconnective tissue, grossly encapsulated by a fibrous capsule with thin rim of ossification at the edge of the tumor. The inner surface of the skull was concave and cracked with a central irregularly shaped defect, measuring $3.8 \times 3.1 \mathrm{~cm}$, with protruding soft tissue lesion in continuity with the external component of the mass (Fig. 3). The inked margins of the resected mass were negative for tumor. The cut surface of the tumor was tan-white, fleshy, and glistening with scant focal areas of hemorrhage. The surrounding normal appearing bone was unremarkable; there was no evidence of satellite lesions. The resected dura showed microscopic foci of adherent tumor without evidence of transgression or invasion. Histopathological analysis (Fig. 4) revealed a lobulated spindle cell neoplasm in chondromyxoid matrix with a characteristic ossifying rim. There was no significant necrosis. However, moderate nuclear pleomorphism and atypia were apparent, and there were scattered giant cells and numerous mitotic figures (40 per 50 high-power fields [HPFs]), consistent with a diagnosis of malignant OFMT. Immunohistochemical stains were negative for S100, CD34, Desmin, Actin smooth muscle, and CKAE1/AE3. Staining was also negative for STAT6/ MUC4. INI1 showed retained nuclear expression throughout the lesional tissue. There was patchy weak staining for CD99.

The postoperative course was unremarkable; the patient was monitored in the neuroscience intensive care unit overnight, transferred to the general neurosurgical ward on postoperative day 1 , and discharged home on the morning of postoperative day 2. A
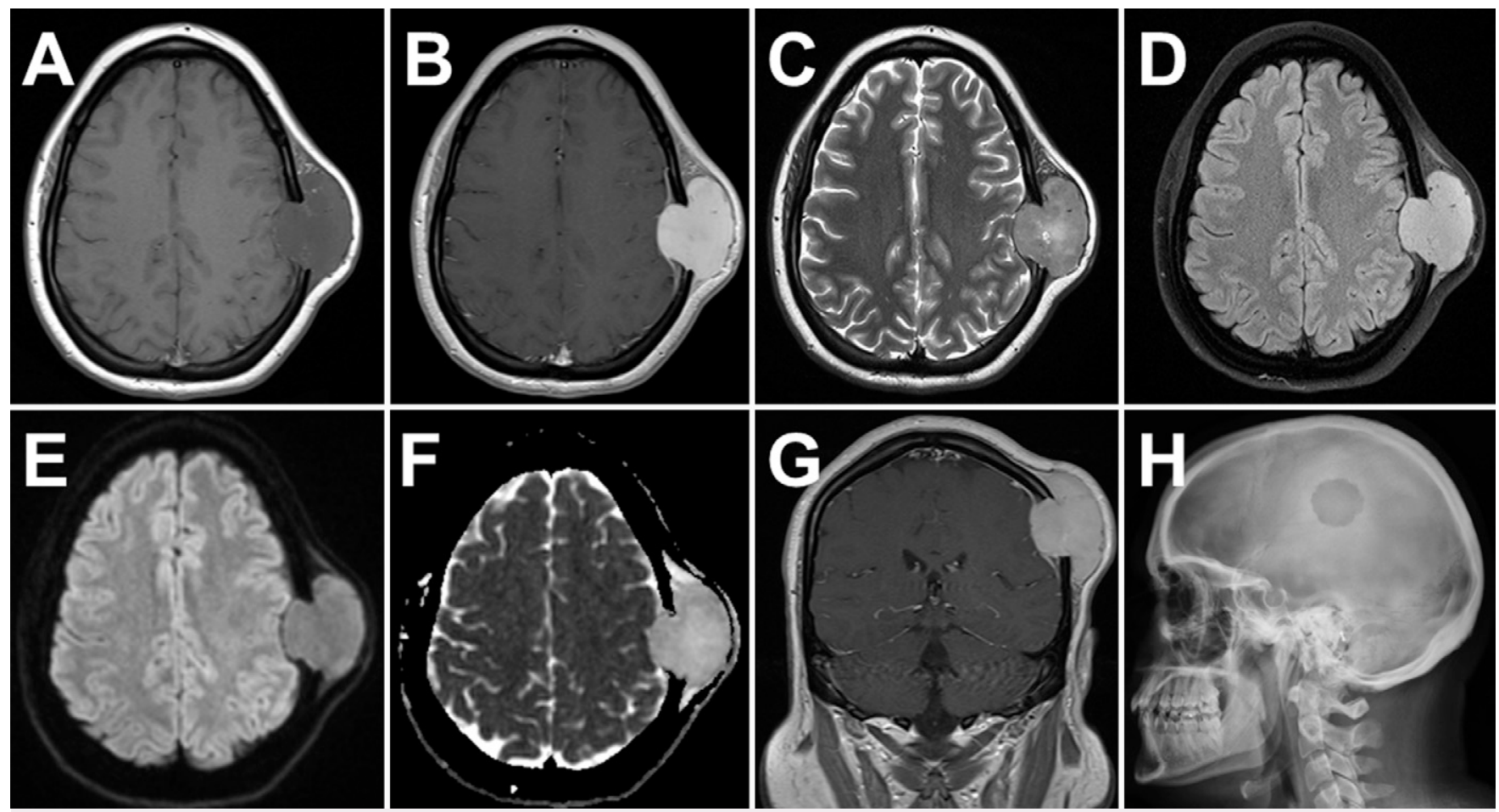

FIG. 1. Preoperative imaging showing $5 \mathrm{~cm}$ by $3.8 \mathrm{~cm}$ by $5.6 \mathrm{~cm}$ transcranial left parietal mass with intra- and extracranial extension and central lytic destruction of the calvarial bone with approximately 1-cm dural/cortical displacement. A: Axial T1-weighted without contrast. B: Axial T1-weighted with contrast. C: Axial T2-weighted. D: Axial fluid-attenuated inversion recovery. E: Axial diffusion weighted imaging. F: Axial apparent diffusion coefficient. G: Coronal T1-weighted with contrast. H: Lateral skull radiograph showing a 3-cm radiolucent lesion at the left parietal calvaria. 


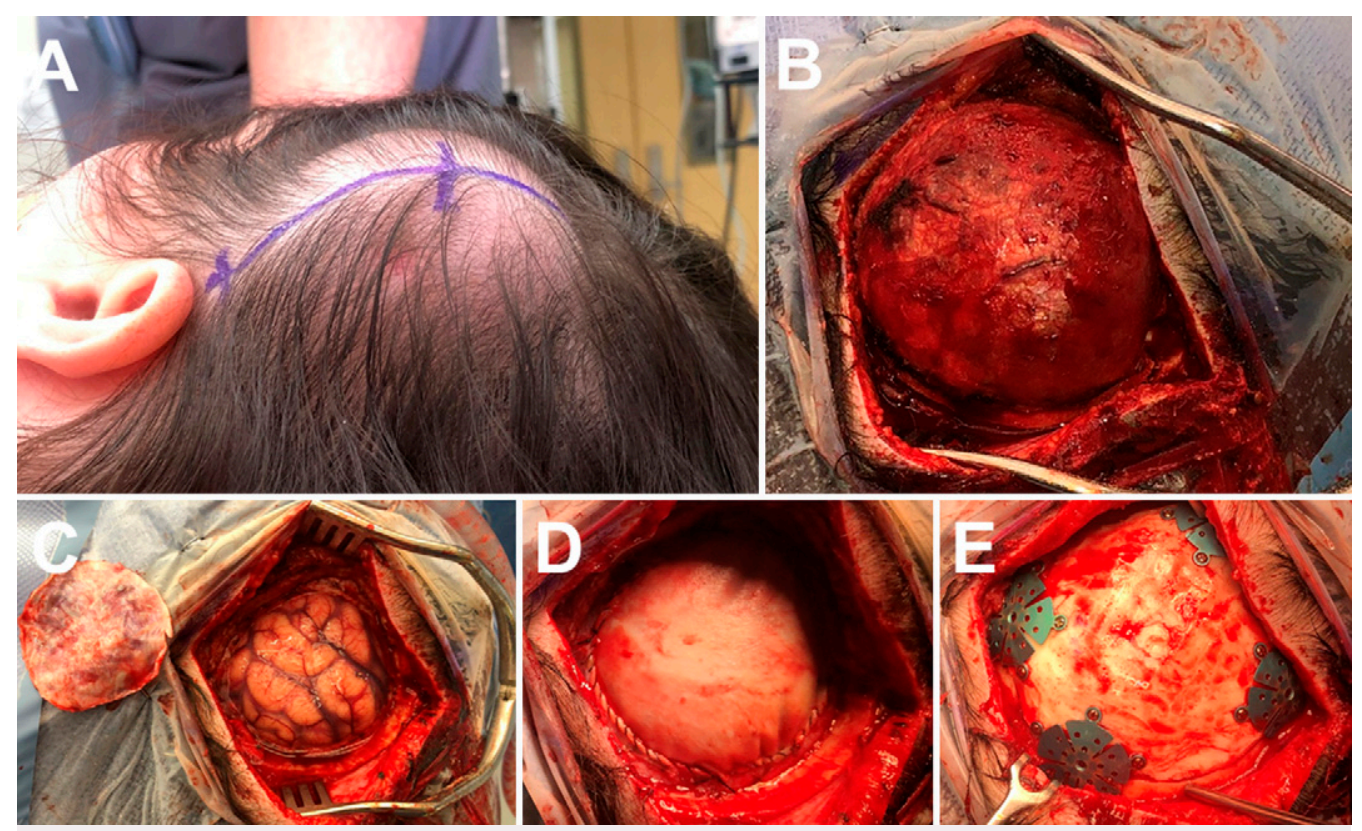

FIG. 2. Surgical approach. A: Left parietal linear incision overlying the soft tissue mass with careful attention not to violate the capsule. B: External surface of the mass capsule with circumferential exposure of surrounding bony margins. C: Underlying cortical surface after en bloc craniectomy gross total resection and dural excision without evidence of brain invasion. D: Dural allograft. E: In situ methylmethacrylate cranioplasty with cranial plating system.

postoperative MRI without and with contrast was obtained on postoperative day 1 that showed complete resection without evidence of residual enhancement. Computed tomography (CT) of the chest and abdomen/pelvis with contrast were obtained that showed no evidence of metastatic disease. The patient is being followed with serial surveillance imaging without any evidence of recurrence.

\section{Discussion}

\section{Observations}

The tumor presented herein showed morphologically classical appearance with dense fibroconnective tissue encapsulated by a fibrous capsule with an ossified rim. There was central destruction of the skull. Measuring $5 \mathrm{~cm}$ by 3.8 by $5.6 \mathrm{~cm}$, the mass was adherent to the underlying dura causing approximately $1 \mathrm{~cm}$ of cortical/dural depression without any macro- or microscopic evidence of dural or brain invasion. Immunohistochemistry was negative for S100, CD34, desmin, smooth muscle actin, cytokeratin, STAT6/MUC4; INI1 was retained. The tumor presented in this report showed 40 mitoses per 50 HPFs, which is along the upper spectrum reported in the literature. The tumor also showed a moderate degree of nuclear atypia, but no necrosis was observed.

\section{Lessons}

OFMTs affect predominantly young to middle-aged adults with a slight male predilection; however, they can occur at any age, and a wide range has been documented ( 3 weeks to 83 years). ${ }^{13,24}$ It most commonly presents as a gradually enlarging circumscribed soft tissue mass. While typically painless, occasional reports of pain and/or paresthesias have been described. ${ }^{33}$ The clinical course is commonly described as a slowly enlarging lesion often over multiple
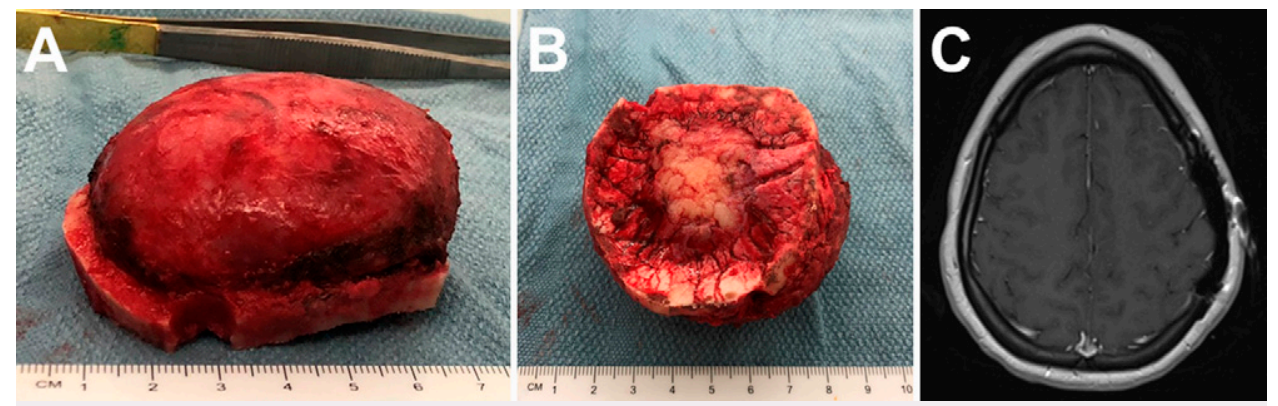

FIG. 3. Gross specimen after en bloc oncological resection. A: Lateral view of the tumor capsule and circumferential craniectomy margin with longitudinal diameter. B: Inferior surface of the specimen showing transcranial growth with calvarial central lytic bony defect and transverse diameter. C: Postoperative T1-weighted MRI with contrast showing gross total resection without evidence of residual contrast enhancement. 

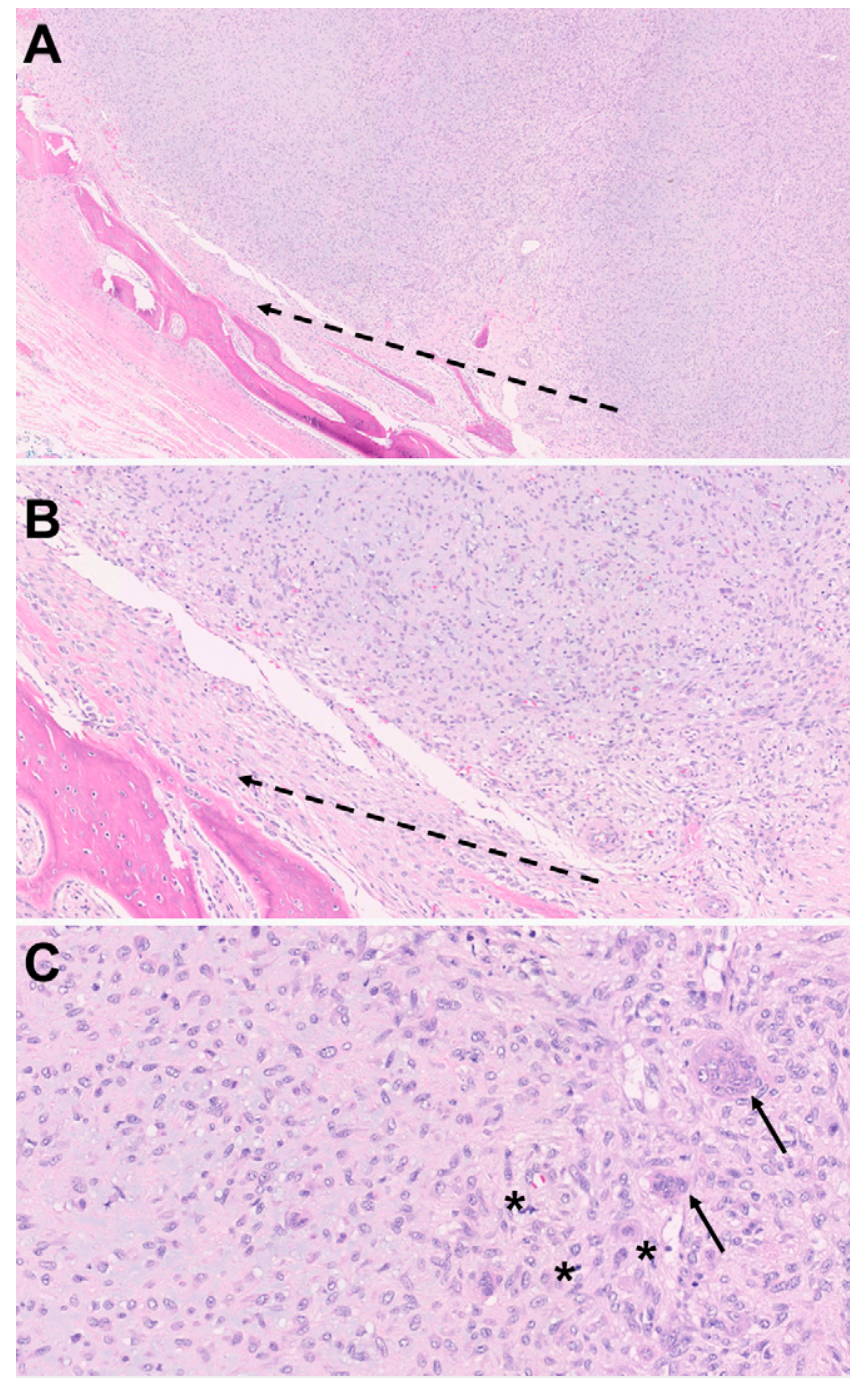

FIG. 4. Histopathology (hematoxylin and eosin stain). A: Original magnification $\times 3$ 3.3. B: Original magnification $\times 10.5$. C: Original magnification $\times 40$. Lobulated spindle cells in chondromyxoid matrix; moderate nuclear pleomorphism and atypia. Dashed arrows indicate the peripheral rim of hypocellular ossification forming incomplete shell of capsular lamellar bone, solid arrows indicate scattered giant cells, and asterisks indicate frequent mitoses.

years (range 1-20 years), ${ }^{13}$ and lesions have been reported at varying sizes, ranging from 1 to $21 \mathrm{~cm}^{6,10,13}$ at presentation. Local recurrence rates have been reported in up to $22 \%$ of patients across the histological spectrum, with a typical interval around 10 years from index resection. ${ }^{19}$ Frequency of metastatic spread has been estimated at $5 \%$ of cases and more common in cases of malignant variants. ${ }^{6,10}$ Imaging appearance on MRI can be variable, commonly isointense on T1-weighted and iso- to hyperintense on T2-weighted; focal signal irregularities can represent areas of hemorrhage or calcification. Leading differential diagnoses by imaging appearance include meningioma, solitary fibrous tumor, gliosarcoma, other sarcoma entities, and metastases. Classically, on CT imaging, OFMTs demonstrate a characteristic thin rim of peripheral ossification in approximately two-thirds of cases. ${ }^{13}$ Morphologically, OFMT presents as a dense fibrous, white to grayish, fleshy, firm to rubbery circumscribed mass. A small peripheral rim of hypocellular ossification/osteoid deposition is characteristic for the majority of cases, forming an incomplete shell of capsular mature lamellar bone. ${ }^{1,6}$ Small focal intratumoral calcifications are also possible, and nonossifying variants occur. Notable differential diagnoses include other soft tissue sarcomas such as low-grade fibromyxoid sarcoma, sclerosing epithelioid fibrosarcoma, synovial sarcoma, Ewing sarcoma, osteosarcoma, chondrosarcoma, and malignant peripheral nerve sheath tumor but also ossifying epithelioid hemangioendothelioma or ossifying hematoma.

Histologically, OFMT is characterized by loose fibromyxoid stroma with dense lobules of relatively uniform polygonal spindle cells in nests, cords, or trabeculae with fibrous septations. The cells are characterized by distinct cell membranes, variably scant eosinophilic cytoplasm, and round to oval vesicular nuclei with conspicuous nucleoli. OFMT is frequently positive for $\mathrm{S} 100$ and is more commonly expressed in typical than atypical or malignant variants ( $88 \%$ versus $75 \%$ versus $42 \%$, respectively, in one study $\left.{ }^{10}\right)$. Similarly, desmin is commonly expressed, staining positive in approximately half of typical/atypical cases and approximately one-quarter of malignant variants. ${ }^{10}$ Additionally, OFMT can variably express GFAP, vimentin, smooth muscle actin, cytokeratins, epithelial membrane antigen, neurofilament, CD56, or collagen II. $3,6,10,13,31,33$ Loss of INI-1 (commonly in mosaic pattern) or overexpression of EAAT4 and MUC4 are also not infrequent. ${ }^{10}$

While there are no uniformly recognized histological criteria for malignancy, a three-tiered classification system has been proposed: typical, atypical, and malignant. ${ }^{6,19}$ Atypical features including hypercellularity, nuclear atypia, infiltrative growth pattern, and elevated mitotic activity have shown statistically significant correlations with clinically more aggressive courses, including elevated risk for local recurrence ${ }^{19}$ and, less commonly, distant metastatic spread. ${ }^{6,33}$ The presence of mitotic activity is highly variable, and in large series, has been reported as ranging from 0 to 40 per 50 HPF. $^{6,19}$ For classification as malignant OFMT, a cutoff of $>12$ mitoses per 50 HPFs has been proposed. ${ }^{6,19}$ Focal necrosis, while not characteristic, can be observed in $10 \%{ }^{19}$ to less than $20 \%{ }^{6}$ of cases; vascular invasion occurs in approximately $10 \%$ of cases. ${ }^{6}$ Similar to other sarcoma entities, molecular associations and signature mutations continue to emerge: certain recurrent gene rearrangements involving the PHF1 gene have been reported, notably EP400-PHF1 translocation fusion genes. In a series of 41 OFMTs, Graham et al. detected PHF1 gene rearrangements in $49 \%$ of tumors, including $43 \%$ typical, $50 \%$ atypical, and $52 \%$ malignant variants. $^{20}$ In a similar immunohistopathological cohort study of 39 OFMTs, Antonescu et al. identified additional recurrent gene fusions, increasing the yield to $85 \%$ of tumor samples (33 of 39 tested tumors). ${ }^{34}$ Additionally, INI-1/SMARCB1 deletions have been implicated in the pathogenesis of various sarcoma subtypes and have recently also been detected in a subset of OFMTs. ${ }^{10}$

Herein, we present a rare case of malignant OFMT with primary skull involvement and transcranial extension, which, to our knowledge, has not previously been reported. No evidence of metastatic disease was apparent at the time of the index operation. Generally, treatment consists of gross total resection, favored en bloc, and complete excision is generally considered curative. Routine adjuvant radiotherapy is generally not recommended. Close follow-up is prudent, especially for atypical or malignant variants that should be regarded as sarcomas with potential for local recurrence on metastatic spread. 


\section{References}

1. Enzinger FM, Weiss SW, Liang CY. Ossifying fibromyxoid tumor of soft parts. A clinicopathological analysis of 59 cases. Am J Surg Pathol. 1989;13(10):817-827.

2. Schofield JB, Krausz T, Stamp GW, Fletcher CD, Fisher C, Azzopardi JG. Ossifying fibromyxoid tumour of soft parts: immunohistochemical and ultrastructural analysis. Histopathology. 1993;22(2):101-112.

3. Williams SB, Ellis GL, Meis JM, Heffner DK. Ossifying fibromyxoid tumour (of soft parts) of the head and neck: a clinicopathological and immunohistochemical study of nine cases. J Laryngol Otol. 1993;107(1):75-80.

4. Kilpatrick SE, Ward WG, Mozes M, Miettinen M, Fukunaga M, Fletcher CD. Atypical and malignant variants of ossifying fibromyxoid tumor. Clinicopathologic analysis of six cases. Am J Surg Pathol. 1995;19(9):1039-1046.

5. Zámecník M, Michal M, Simpson RH, Lamovec J, Hlavcák P, Kinkor $Z$, et al. Ossifying fibromyxoid tumor of soft parts: a report of 17 cases with emphasis on unusual histological features. Ann Diagn Pathol. 1997;1(2):73-81.

6. Folpe AL, Weiss SW. Ossifying fibromyxoid tumor of soft parts: a clinicopathologic study of 70 cases with emphasis on atypical and malignant variants. Am J Surg Pathol. 2003;27(4):421-431.

7. Suehiro K, Pritzwald-Stegmann P, Lee KM, Teoh HH, Alison PM. Mediastinal and pulmonary metastases of malignant ossifying fibromyxoid tumor. Ann Thorac Surg. 2006;81(6):2289-2291.

8. Cibull TL, Billings SD. Cutaneous malignant ossifying fibromyxoid tumor. Am J Dermatopathol. 2007;29(2):156-159.

9. Sangala JR Jr, Park P, Blaivas M, Lamarca F. Paraspinal malignant ossifying fibromyxoid tumor with spinal involvement. J Clin Neurosci. 2010;17(12):1592-1594.

10. Graham RP, Dry S, Li X, Binder S, Bahrami A, Raimondi SC, et al. Ossifying fibromyxoid tumor of soft parts: a clinicopathologic, proteomic, and genomic study. Am J Surg Pathol. 2011;35(11): $1615-1625$

11. Tanna N, Chadha N, Sharma RR, Goodman JF, Sadeghi N. Malignant ossifying fibromyxoid tumor of the parapharyngeal space. Ear Nose Throat J. 2012;91(10):E15-E17.

12. Ohta K, Taki M, Ogawa I, Ono S, Mizuta K, Fujimoto S, et al. Malignant ossifying fibromyxoid tumor of the tongue: case report and review of the literature. Head Face Med. 2013;9(16):16.

13. Bakiratharajan D, Rekhi B. Ossifying Fibromyxoid Tumor: An Update. Arch Pathol Lab Med. 2016;140(4):371-375.

14. Dantey K, Schoedel K, Yergiyev O, McGough R, Palekar A, Rao UNM. Ossifying fibromyxoid tumor: a study of 6 cases of atypical and malignant variants. Hum Pathol. 2017;60:174-179.

15. Donner LR. Ossifying fibromyxoid tumor of soft parts: evidence supporting Schwann cell origin. Hum Pathol. 1992;23(2):200-202.

16. Matsumoto K, Yamamoto T, Min W, Yamada N, Asano G, Moriyama $\mathrm{M}$, Matsumoto T. Ossifying fibromyxoid tumor of soft parts: clinicopathologic, immunohistochemical and ultrastructural study of four cases. Pathol Int. 1999;49(8):742-746.

17. Ekfors TO, Kulju T, Aaltonen M, Kallajoki M. Ossifying fibromyxoid tumour of soft parts: report of four cases including one mediastinal and one infantile. APMIS. 1998;106(12):1124-1130.

18. Hirose T, Shimada S, Tani T, Hasegawa T. Ossifying fibromyxoid tumor: invariable ultrastructural features and diverse immunophenotypic expression. Ultrastruct Pathol. 2007;31(3):233-239.

19. Miettinen M, Finnell V, Fetsch JF. Ossifying fibromyxoid tumor of soft parts-a clinicopathologic and immunohistochemical study of 104 cases with long-term follow-up and a critical review of the literature. Am J Surg Pathol. 2008;32(7):996-1005.

20. Graham RP, Weiss SW, Sukov WR, Goldblum JR, Billings SD, Dotlic S, Folpe AL. PHF1 rearrangements in ossifying fibromyxoid tumors of soft parts: A fluorescence in situ hybridization study of 41 cases with emphasis on the malignant variant. Am J Surg Pathol. 2013;37(11):1751-1755.

21. Williams RW, Case CP, Irvine GH. Ossifying fibromyxoid tumour of soft parts-a new tumour of the parotid/zygomatic arch region. $\mathrm{Br} \mathrm{J}$ Oral Maxillofac Surg. 1994;32(3):174-177.

22. Ng WK. Pathologic quiz case 2. Ossifying fibromyxoid tumor. Arch Otolaryngol Head Neck Surg. 1995;121(11):1321-1323.

23. Thompson J, Castillo M, Reddick RL, Smith JK, Shockley W. Nasopharyngeal nonossifying variant of ossifying fibromyxoid tumor: CT and MR findings. AJNR Am J Neuroradiol. 1995;16(5):1132-1134.

24. Al-Mazrou KA, Mansoor A, Payne M, Richardson MA. Ossifying fibromyxoid tumor of the ethmoid sinus in a newborn: report of a case and literature review. Int J Pediatr Otorhinolaryngol. 2004;68(2):225-230.

25. Blum A, Back W, Naim R, Hörmann K, Riedel F. Ossifying fibromyxoid tumor of the nasal septum. Auris Nasus Larynx. 2006;33(3): 325-327.

26. Mollaoglu N, Tokman B, Kahraman S, Cetiner S, Yucetas S, Uluoglu $O$. An unusual presentation of ossifying fibromyxoid tumor of the mandible: a case report. J Clin Pediatr Dent. 2006;31(2): 136-138.

27. Miliaras D, Meditskou S, Ketikidou M. Ossifying fibromyxoid tumor may express CD56 and CD99: a case report. Int J Surg Pathol. 2007;15(4):437-440.

28. Sharif MA, Mushtaq S, Mamoon N, Khadim MT. Ossifying fibromyxoid tumor of oral cavity. J Coll Physicians Surg Pak. 2008;18(3): 181-182.

29. Nonaka CF, Pacheco DF, Nunes RP, Freitas RdeA, Miguel MC. Ossifying fibromyxoid tumor in the mandibular gingiva: case report and review of the literature. J Periodontol. 2009;80(4):687-692.

30. Kondylidou-Sidira A, Kyrgidis A, Antoniades H, Antoniades K. Ossifying fibromyxoid tumor of head and neck region: case report and systematic review of literature. J Oral Maxillofac Surg. 2011;69(5): 1355-1360.

31. Velasco IA, Zhang R, Li T, Wang D. Ossifying Fibromyxoid tumor of soft parts in head and neck: case report and literature review. Diagn Pathol. 2018;13(1):21.

32. Seykora JT, Kutcher C, van de Rijn M, Dzubow L, Junkins-Hopkins $\mathrm{J}$, loffreda M. Ossifying fibromyxoid tumor of soft parts presenting as a scalp cyst. J Cutan Pathol. 2006;33(8):569-572.

33. Schneider N, Fisher C, Thway K. Ossifying fibromyxoid tumor: morphology, genetics, and differential diagnosis. Ann Diagn Pathol. 2016;20:52-58.

34. Antonescu CR, Sung YS, Chen CL, Zhang L, Chen HW, Singer S, et al. Novel ZC3H7B-BCOR, MEAF6-PHF1, and EPC1-PHF1 fusions in ossifying fibromyxoid tumors-molecular characterization shows genetic overlap with endometrial stromal sarcoma. Genes Chromosomes Cancer. 2014;53(2):183-193.

\section{Disclosures}

The authors report no conflict of interest concerning the materials or methods used in this study or the findings specified in this paper.

\section{Author Contributions}

Conception and design: both authors. Acquisition of data: both authors. Analysis and interpretation of data: both authors. Drafting the article: both authors. Critically revising the article: both authors. Reviewed submitted version of manuscript: both authors. Administrative/technical/ material support: Graham. Study supervision: Graham.

\section{Correspondence}

R. Scott Graham: Virginia Commonwealth University Health System, Richmond, VA. robert.graham@vcuhealth.org. 